\title{
Factors predicting overall success: a review of 747 microsurgical vasovasostomies
}

\author{
Stéphane Bolduc, MD; Marc Anthony Fischer, MD; Genevieve Deceuninck, MSC; Michel Thabet, MD
}

\begin{abstract}
Objectives: Advances in surgical techniques have improved the outcome of microsurgical vasovasostomy (VV). We performed a retrospective analysis of surgical procedures to determine outcomes and predictors of $\mathrm{V} V$ success, to develop Kaplan-Meier Curves for predicting VV outcomes and to evaluate the use of $\alpha$-glucosidase (AG) to predict outcomes.
\end{abstract}

Patients and Methods: We undertook a retrospective analysis of 747 modified 1-layer microsurgical VV procedures performed between 1984 and 2000. Obstructive interval, partner status, social status preoperatively and method of vasal obstruction, vasal fluid quality and sperm granuloma intraoperatively were compared with outcome results. Parameters evaluated at follow-up included semen analysis, AG concentration in ejaculate fluid and pregnancy rates.

Results: The overall patency rate was $86 \%$ and pregnancy rates were $33 \%$ and $53 \%$ at 1 and 2 years after primary VV, respectively. Preoperative factors associated with successful outcome and pregnancy included shorter obstructive interval and same female partner $(p<0.05)$. Intraoperative factors predicting success included the use of surgical clips instead of suture at vasectomy, the presence of a sperm granuloma, the presence and quality of vasal fluid, and the presence and quality of sperm in vasal fluid. Further, increased AG in the postoperative semen predicted improved patency and pregnancy outcomes.

Conclusion: This study confirms the effectiveness of $\mathrm{V} V$ for vasectomized men who wish to father children. It also demonstrates that preoperative and intraoperative factors are predictive of the VV outcome. Postoperative AG is also a useful marker of patency and it appears to predict pregnancy outcome.

CUAJ 2007;1(4):388-94

\section{Introduction}

Vasectomy is a simple and reliable method of permanent contraception that has achieved widespread acceptance. The popularity of the operation combined with increasing marital and relationship separation has resulted in a growing number of men requesting vasectomy reversal. ${ }^{1,2}$ There are studies demonstrating acceptable patency and pregnancy rates of vasovasostomy (VV) without optical magnification ${ }^{3,4}$ and improved success rates with optical loupe assisted VV.5,6 Modern microsurgical techniques, as popularized by Silber, ${ }^{7}$ remain the standard with which all other methods of vasectomy reversal are compared. Patency is over $80 \%$ in most microsurgical series, with patency approaching $100 \%$ in some reports. ${ }^{3,7-13}$ However, prediction of patency and preg- nancy is based primarily on evaluation of preoperative and intraoperative factors as well as semen analysis.

$\alpha$-glucosidase (AG) is an epididymal secretory product that can serve as an indicator of epididymal function. Normal or increased AG levels have been associated with improved outcomes of in vitro fertilization. It has previously been shown that $\alpha-1-4$-glucosidase can be used to assist in the etiologic diagnosis of azoospermia. ${ }^{14-16}$ We sought to define the utility of measuring AG as a prognostic indicator for successful vasectomy reversal. AG concentration determined in postoperative ejaculate of men who underwent $\mathrm{V} V$ may predict both patency and pregnancy outcomes following VV; AG may also be used as a prognostic indicator for counselling patients postoperatively about the success of VV. The utility of measuring this enzyme to assist in the prediction of outcome has not been assessed in previous $\mathrm{VV}$ studies.

The goal of this study is to compare the outcomes, including patency and pregnancy rates, following $\mathrm{VV}$ and to provide an understanding of factors involved in the surgery that may predict outcomes of VV. Further, we sought to develop an understanding of the utility of AG in predicting the success in achieving pregnancy after $\mathrm{VV}$.

\section{Patients and Methods}

We retrospectively reviewed 747 consecutive $\mathrm{VV}$ procedures performed by a single surgeon between January 1984 and December 2000. All surgeries were performed using the modified 1-layer microsurgical technique with 4 to 6 full thickness 9-0 sutures and 9-0 interposed sutures through the muscularis and adventicia. ${ }^{9}$ Criteria for inclusion in the study were a minimum of 3 months of follow-up 
with semen analysis (performed according to WHO methods ${ }^{17}$ ) including the measurement of AG in ejaculate. Patients were excluded if they did not provide a semen analysis (101 patients) or if they did not attempt to achieve a pregnancy (41 patients). The remaining 605 patients (81\%) provided a minimum of 1 semen analysis; all of the samples were analyzed at the same andrology laboratory within our institution, which has previously reported on the use of AG in men with azoospermia. ${ }^{15,18}$ Demographic data included patient's age at vasectomy and $\mathrm{VV}$, the obstructive interval between vasectomy and $\mathrm{VV}$, prior paternity status, partner status (same or new partner) and the age of the female partner.

Measurement of seminal plasma AG specific activity was performed with paranitrophenyl- $\alpha$-Dglucosidase (PNPG) as substrate (Sigma Chemical Company, St. Louis, Mo.) according to a previously published method..$^{18}$ Antisperm antibodies (IgA, $\lg \mathrm{G}$ and $\lg M$ ) were detected and quantified by immunobeads assay (IBT). ${ }^{19}$

At the time of surgery, the method of vasal occlusion (clip or ligature), the presence of sperm granuloma (confirmed by histological examination), the presence of vas fluid from the testicular end and sperm content of vasal fluid (microscopic examination) were recorded. The gross appearance of the vasal fluid was rated as clear, opalescent or creamy-thick. The presence of sperm in the vasal fluid was graded as follows:

- Grade 1: motile normal sperm.

- Grade 2: non-motile normal shaped sperm.

- Grade 3: non-motile sperm, predominately sperm heads (no tails) with some normal shaped sperm.

- Grade 4: exclusively sperm heads.

- Grade 5: no sperm. ${ }^{20}$

Semen analysis was recommended at 3-month intervals or until establishment of pregnancy, or 2-year follow up. Patency was defined as the presence of motile sperm in the ejaculate in at least 1 analysis. Pregnancy outcomes were obtained at either follow-up visits or via telephone interviews. Pregnancy was defined as unassisted establishment (no assisted reproduction) of a viable pregnancy leading to live birth.

Statistical analyses were performed with chisquared tests, logistic regression and multivariate analysis, as appropriate, using SAS System 6.11 (SAS Institute Inc., Cary, NC). Patency and preg- nancy rates were correlated to the obstructive intervals and Kaplan-Meier curves were established.

\section{Results}

\section{Patient demographics}

In the present study of 605 men, 559 (92.4\%) underwent primary $\mathrm{VV}$ and $46(7.6 \%)$ had a repeat VV (secondary) after a failed previous attempt. Among the 46 patients who underwent secondary VV, $15(33 \%)$ had initially established patency following the initial VV procedure and 2 couples had a pregnancy. These men then went on to become azoospermic and requested another attempt at $\mathrm{VV}$.

Men were engaged in the same marital relationship after vasectomy in 193 cases (32\%) and the remaining 412 patients (68\%) had new partners. The mean age at vasectomy was 30.3 years (standard deviation [SD] $4.6 \mathrm{yr}$ ) and 37.2 years (SD $5.4 \mathrm{yr}$ ) at VV. Mean obstructive interval was 82 months (SD $47 \mathrm{mo}$ ). Most patients (93\%) had fathered at least 1 child before vasectomy. Mean female partner age was 29.9 years (SD $4.7 \mathrm{yr}$ ). Overall, 338 (55.9\%) of the spouses had not been pregnant before consultation.

\section{Surgery and patency}

Intraoperative parameters were recorded and correlated to patency and pregnancy. Significant observations are presented in Table 1. For the 559 cases of primary VV, 531 (95\%) were performed bilaterally; $30(65 \%)$ of the second VVs were bilateral.

At least 1 semen analysis was provided by each of the 605 patients. Overall mean results for individual parameters are shown in Table 2. Overall, patency was confirmed by semen analysis in 491 men $(87.8 \%)$ after the first $\mathrm{VV}$ and in 27 (59\%) of those who underwent a second attempt at vasectomy reversal. The patency rate in both primary and secondary procedures correlated with a bilateral VV (odds ratio [OR] 2.8, 95\% confidence interval $[\mathrm{Cl}] 1.2-6.4, p=0.013)$.

Normal semen parameters, according to $\mathrm{WHO}$ criteria, were noted in 300 (58\%) patent men. The remaining 218 men had oligospermia or asthenospermia with or without teratospermia. Azoospermia persisted in $68(12.2 \%)$ and $19(41 \%)$ men after a primary and a repeat $\mathrm{VV}$, respectively. 
Reduced semen quality as well as patency correlated with the obstructive interval $(p=0.005)$ (Fig. 1).

\section{Pregnancy}

Pregnancies after primary VV were achieved after 1 year by 145 (33.4\%) couples and by 198 (52.9\%) couples at 2 years. A further 19 couples achieved pregnancy after 2 years. Following a secondary VV, $11(30 \%)$ couples achieved a pregnancy within 2 years. Overall, the mean time to achieve pregnancy was 10.7 months (range 1-36 mo) after both primary and secondary $\mathrm{VV}$.

Pregnancy rates in both groups correlated with the status of the female partner (same partner and whether she had previously been pregnant) (Table 1). Pregnancy rates also correlated with the obstructive interval $(p<0.001)$ (Fig. 2) but did not correlate with unilateral or bilateral VV.

\section{$\alpha$-glucosidase}

The mean AG level was 33 mU/ejaculate (SD $5 \mathrm{mU} / \mathrm{ejaculate)}$ for azoospermic patients and $106 \mathrm{mU} /$ ejaculate (SD $41 \mathrm{mU} /$ ejaculate) for patent patients (count $>5 \times 10^{6} / \mathrm{mL}, p<0.001$ ). There was a correlation between the level of AG and the sperm count (Fig. 3) and motility (Fig. 4). We noted a significant association between lower levels of AG and oligospermia $(p<0.02)$ as well as asthenospermia $(p<0.003)$. The duration of vasal obstruction was

Table 1: Significant associations between preoperative, intraoperative and postoperative factors and the success of vasectomy reversal

\begin{tabular}{|c|c|c|c|}
\hline Correlations & Observations & OR $(95 \% \mathrm{Cl})$ & $p$ value \\
\hline Type of ligature at vasectomy v. patency & Clips: 94\%; suture: $83.4 \%$ & $3.1(1.5-6.6)$ & 0.006 \\
\hline Sperm granuloma (36.4\%) v. patency & Presence: 93\%; absence: $84.6 \%$ & $2.4(1.3-4.4)$ & 0.004 \\
\hline Granuloma v. type of vas fluid & $\begin{array}{l}\text { Opalescent-clear: } 94 \% \text {; } \\
\text { creamy-thick: } 80 \%\end{array}$ & $1.6(1.2-2.1)$ & $<0.001$ \\
\hline Granuloma v. sperm in vas fluid & Sperm: $90 \%$; no sperm: $75 \%$ & $1.5(1.1-2.0)$ & 0.002 \\
\hline Granuloma v. sperm grade & Grade $1>2>3-4$ & NS & $<0.001$ \\
\hline Type of vas fluid $v$. presence of sperm & $\begin{array}{l}\text { Opalescent: } 89 \% \text { > clear: } 80 \% \\
\text { > creamy-thick: } 75 \%\end{array}$ & NS & $<0.001$ \\
\hline Sperm in vas fluid (91\%) v. patency rate & Sperm: $85 \%$; no sperm: $66 \%$ & $5.1(2.6-9.8)$ & $<0.001$ \\
\hline Sperm in vas fluid v. pregnancy rate & Sperm: $38 \%$; no sperm: $16 \%$ & $3.2(1.3-8.0)$ & $<0.004$ \\
\hline Obstructive interval v. patency rate & $\begin{array}{l}\text { Decrease patency rate with time; } \\
\text { patent: } 6.5 \mathrm{yr} \text {, azoospermia: } 8.8 \mathrm{yr}\end{array}$ & $0.91 / y r(0.88-0.96)$ & 0.005 \\
\hline Obstructive interval v. pregnancy rate & $\begin{array}{l}\text { Decrease pregnancy rate with time; } \\
\text { pregnancy: } 5.7 \mathrm{yr} \text {, no pregnancy } 7.2 \mathrm{yr}\end{array}$ & $0.91 / y r(0.87-0.96)$ & 0.003 \\
\hline Same partner v. pregnancy rate & Same: $45 \%$; new: $30.7 \%$ & $1.8(1.2-2.5)$ & $<0.002$ \\
\hline Previous pregnancy $v$. pregnancy rate & Yes: $41 \%$; no: $31 \%$ & $1.5(1.1-2.2)$ & $<0.02$ \\
\hline \multirow[t]{3}{*}{ Semen analysis* v. pregnancy rate } & Patent v. azoospermic & $5.6(3.2-9.8)$ & $<0.001$ \\
\hline & Normal: 44\%; anomalies: $23 \%$ & $5.5(1.4-23)$ & $<0.001$ \\
\hline & Anomalies: 23\%; azoospermia 3.3\% & $4.6(1.2-19)$ & $<0.001$ \\
\hline
\end{tabular}

Table 2: Mean semen parameters at follow-up for patent patients following vasovasostomy

\begin{tabular}{lc} 
Parameter & Mean (and SD)* \\
\hline Semen volume, $\mathrm{mL}$ & $2.9(1.3)$ \\
Sperm count per $\mathrm{mL}$ & $31 \times 10^{6}\left(8 \times 10^{6}\right)$ \\
Motility, \% motile & $38(6)$ \\
Morphology, \% normal & $28(9)$ \\
$\alpha$-glucosidase & $88 \mathrm{mU} /$ ejaculate, $30.3 \mathrm{mU} / \mathrm{mL}$ \\
\hline${ }^{*}$ Unless otherwise indicated. &
\end{tabular}


inversely correlated with concentration of AG in the ejaculate $(p<0.01)$. Moreover, higher levels of AG were significantly correlated to the success of achieving pregnancy $(p<0.04)$. Three pregnancies were reported for patients with AG levels below $45 \mathrm{mU} /$ ejaculate $(20 \mathrm{mU} / \mathrm{mL}) ; 1$ had severe oligospermia $\left(<5 \times 10^{6} / \mathrm{mL}\right)$ and 2 were azoospermic; all 3 semen analyses were performed after conception. We suspected that these men had established patency but developed worsening semen parameters after establishment of pregnancy.

We specifically analyzed the AG levels of patients undergoing repeat VV. For these 46 men, we observed the trend of the $15(33 \%)$ patients with transient patency. In this group of patients, as sperm counts decreased or patients became azoospermic, the AG level also decreased (data not shown). The mean AG level was $11.4 \mathrm{mU} / \mathrm{ejaculate}$ (SD $5 \mathrm{mU} /$ ejaculate) and $87.5 \mathrm{mU} /$ ejaculate (SD $41 \mathrm{mU} / \mathrm{ejaculate)}$ for azoospermic and patent patients (count $>10 \times 10^{6} / \mathrm{mL}$ ), respectively $(p<0.001)$. At repeat $\mathrm{VV}$, we confirmed obstruction

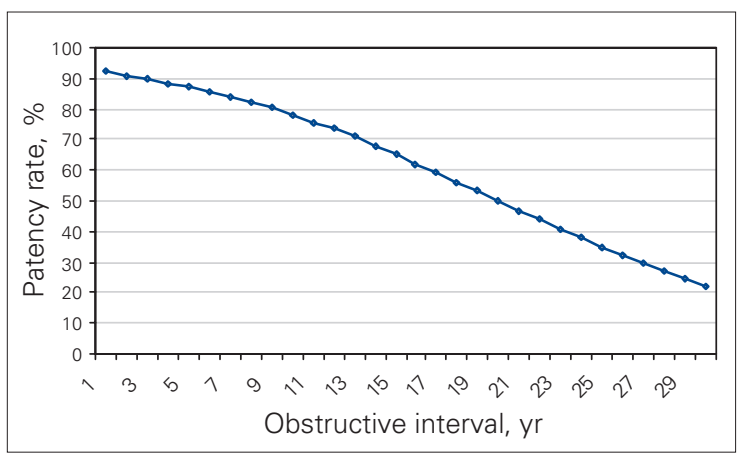

Fig. 1. Patency rate following vasovasostomy, compared with different obstructive intervals, using a Kaplan-Meier curve. There is a significant decrease of patency rates with longer obstructive intervals $(p=0.005)$.

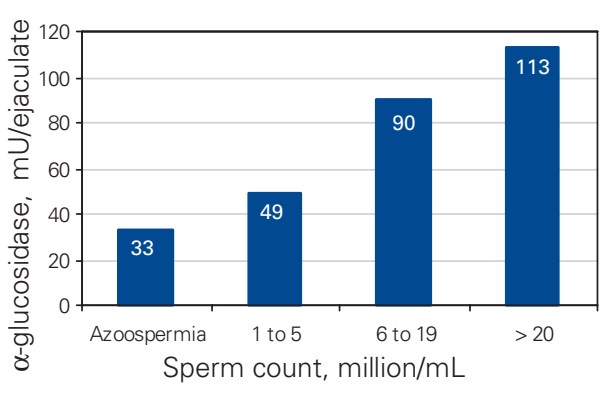

Fig. 3. Seminal $\alpha$-glucosidase concentration (mU/ejaculate) associated with sperm counts (millions $/ \mathrm{mL}$ ). There is a significant difference between each interval $(p<0.02)$. at the first $\mathrm{VV}$ site in 37 of 46 patients $(80 \%)$ by either a sperm granuloma (28 patients) or stenosis (9 patients). Following repeat $\mathrm{VV}$, the AG level of the 27 (59\%) patent patients was comparable to patent patients after primary $\mathrm{VV}$.

\section{Antisperm antibodies}

According to multivariate analysis (Cox regression), there was a significant association between the absence of antisperm antibodies in the seminal fluid following $\mathrm{VV}$ and the success of achieving pregnancy. The absence, compared with the presence, of antisperm antibodies offered a relative risk (RR) of $0.326(95 \% \mathrm{Cl} 0.143-0.745, p=0.003)$. When analyzed at each level of dilution from $1: 2$ to $1: 4096$ it provided an $\mathrm{RR}$ of $0.824(95 \% \mathrm{Cl}$ $0.683-0.995)$ per level $(p=0.04)$. The presence of high titers of antisperm antibodies in the semen decreased the probability of achieving pregnancy.

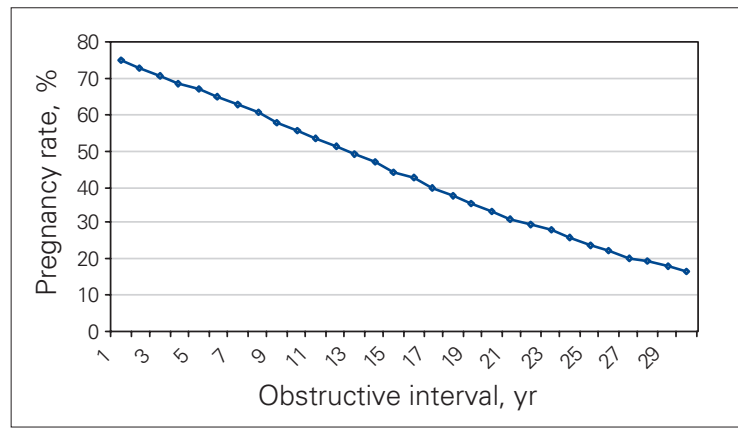

Fig. 2. Pregnancy rate following vasovasostomy, compared with different obstructive intervals using a Kaplan-Meier curve. There is a significant decrease of pregnancy rates with longer obstructive intervals $(p<0.001)$.

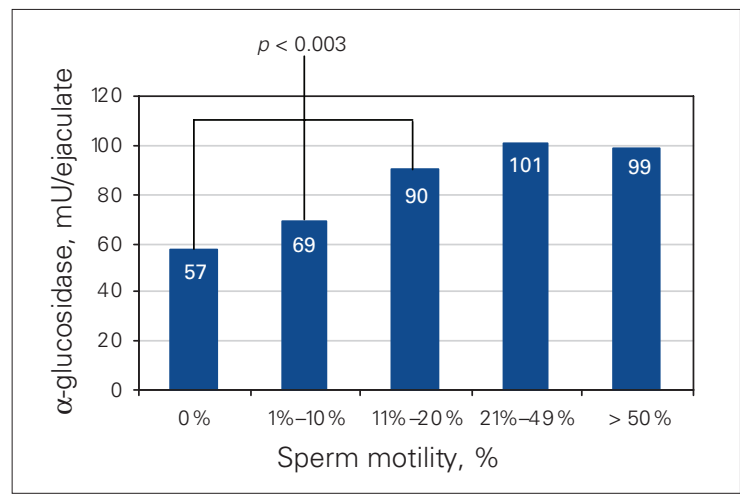

Fig. 4. Seminal $\alpha$-glucosidase concentration (mU/ejaculate) associated with sperm motility (\%) in patent patients. There are significant differences between $0 \%$ motile sperm, $<1 \%-10 \%$ motile sperm and $<11 \%$ motile sperm $(p<0.003)$. 


\section{Discussion}

Most studies report patency, defined as return of sperm to the ejaculate, as the primary outcome measure for VV. Using these criteria, results of series using microsurgical techniques are consistently and reliably superior to series with nonmicrosurgical anastomotic techniques. Patency is over $80 \%$ in most microsurgical series, with some reports approaching $100 \% .^{7-13}$ In our study, we found that microsurgical $\mathrm{VV}$ results in return of sperm in $88 \%$ of men following $\mathrm{VV}$ and the spontaneous pregnancy rate at 2 years of follow up was $53 \%$. It is possible that the pregnancy rate could be improved if couples who were unable to conceive through intercourse were able to access assisted reproductive technologies such as intrauterine insemination, in vitro fertilization or intracytoplasmic sperm injection.

Microsurgical VV is the preferred technique for vasectomy reversal for most urological microsurgeons. The virtues of the main techniques (1-and 2-layer VV) have been debated in the literature. The present series included cases of 1-layer VV. Demonstrable advantages of 1 -layer $\mathrm{VV}$ are a shorter operating time, decreased training time and decreased costs. A theoretical advantage of 1-layer $\mathrm{VV}$ is that fewer sutures pass through the lumen of the vas deferens, which may lower the risk of suture granuloma and stricture at the anastomosis site. Coversely, a formal 2-layer VV provides precise mucosal approximation of the vasal mucosa and a theoretically leak-proof anastomosis. Most studies report similar outcomes of the 2 procedures $^{8}$; thus, 1-layer VV would be adequate for most surgeons. There are particular situations in which a formal 2-layer VV might be preferable to 1-layer VV. For example, if there is a large discrepancy between the diameters of the lumen of the vas to be anastomozed, a 2-layer VV may allow for more accurate anastomosis.

The outcome of vasectomy reversal is influenced by several factors. Studies reporting outcomes of VV consistently demonstrate discrepancies between patency rates and pregnancy rates. The duration of obstruction is a significant factor in the outcome of $\mathrm{VV}$ following vasectomy. Our data are consistent with reported studies demonstrating that pregnancy rate after $\mathrm{VV}$ is inversely related to the duration of vasal obstruction. Vasectomy has time dependent adverse effects on the testis, ${ }^{21}$ epididymis ${ }^{22}$ and vas deferens. ${ }^{20}$ There are immunologic effects that may affect sperm production and activity, although the exact mechanism of development of antisperm antibodies is debatable. However, the detection of these antibodies in almost $80 \%$ of men following vasectomy likely has an impact on pregnancy outcomes and negatively affects future fertility ${ }^{23,24}$ when present in the semen. Our data confirmed these results. Men with higher levels of antisperm antibodies in the semen were significantly less likely to establish a pregnancy compared with men without antisperm antibodies.

In our study, the presence of sperm granuloma was associated with better sperm quality at the anastomosis site and improved outcome, both in terms of patency rate and pregnancy rate. In addition, the presence of sperm in fluid retrieved from the proximal vas deferens before anastomosis was highly correlated with successful patency and pregnancy. Similarly, the quality of vasal fluid, ranging from opalescent to thick and creamy, correlated to successful outcome after VV. These data and similar observations in other studies make it clear that when intravasal azoospermia and thick vasal fluid is encountered at the proximal vas deferens site, it is less likely that sperm will be found in the ejaculate following $\mathrm{VV}(67 \%$ patency in the present series). In these cases, the surgeon could consider performing a vasoepididymostomy rather that going ahead with $\mathrm{VV} .{ }^{8,25}$

A unique observation of our study is the influence of the status of the female partner's fertility on the outcome of VV. Pregnancy rates following successful $\mathrm{VV}$ were higher in couples in which the female partner had previously established a pregnancy, compared with women who had not previously conceived. Female infertility factors may be a cause of the aforementioned discrepancy between successful VV outcome and pregnancy rates. Clearly, surgeons performing VV should be thorough in identifying and helping to facilitate investigation and treatment of possible causes of infertility in the female partner before or in conjunction with $\mathrm{VV}$.

The present study also confirms the success of repeat vasectomy reversal after failed primary repair. The patency and pregnancy rates we report are similar to those reported in other studies. ${ }^{26,27}$ It is hypothesized that most cases of failed primary $\mathrm{VV}$ occur as a result of obstruction at the anas- 
tomosis site. We found that in $37(80 \%)$ of 46 cases of repeat $\mathrm{VV}$, obstruction at the anastomosis site was confirmed with either a sperm granuloma (28 of 46 cases, $61 \%$ ) or a stricture of the vas deferens (9 of 46 cases, $20 \%$ ). Thus it appears that the majority of failures of primary $\mathrm{VV}$ in this series occurred as a result of failured surgical technique. The principles of VV include accurate and leakproof mucosal approximation, a tension-free anastomosis, healthy tissue with a good blood supply and atraumatic anastomosis technique. Observation of these surgical principles can maximize the chance of successful VV. If a previous attempt at VV has failed, the likely causes of failure and the alternative courses of action relevant to the clinical situation should be discussed with the patient. Although assisted reproductive technologies have significantly impacted the treatment of male infertility, microsurgical reconstruction of obstructed genital tracts remains the most successful, rational and cost-effective method of treating men with obstructive azoospermia due to vasectomy. ${ }^{26,27}$

Seminal fluid contains many enzymes, including various hydrolases, which are thought to originate in the accessory reproductive structures, including the epididymis. ${ }^{28}$ It has been previously shown that $\alpha-1-4$-glucosidase disappears from the semen or decreases below $40 \mathrm{mU} /$ ejaculate in men with confirmed vasectomy or genital tract obstruction. ${ }^{14-16}$ However, interpretation of the test is more difficult when the result is not negative. Unilateral obstruction or partial obstruction may exist and account for lower levels in some men. Also, although the cauda epididymis is the primary source of AG, the site of secretion may also include the vas deferens. ${ }^{16}$ We observed that AG is a reliable marker of the success of $\mathrm{VV}$ both in terms of patency and pregnancy rates for patients after primary VV. Further, although patients undergoing a second $\mathrm{V} V$ had an overall lower patency and pregnancy rate than patients undergoing primary repair, the pregnancy rates were equal in the 2 groups if similar amounts of AG were present in the ejaculate. These data indicate that measurement of AG is an important marker of success of both primary and repeat VV. Decreasing sperm count and AG levels after VV leads to the suspicion of either failure of the initial procedure or developing obstruction at the site of the vasal anastomosis. Measurement of AG following $\mathrm{VV}$ may serve as an indicator of which patients may be candidates for repeat VV.

The success of microsurgical $\mathrm{VV}$ in restoring sperm to the ejaculate and in establishing pregnancy make this procedure a successful treatment of choice for men wishing to resume paternity after vasectomy. Assisted reproductive technologies have expanded treatment options for infertile couples. Additional procedures and costs (e.g., drugs and number of surgical procedures) required during assisted reproductive technology are important considerations for couples seeking treatment for obstructive azoospermia following vasectomy. ${ }^{26,27,29,30}$ Decision analysis studies have demonstrated that patency rates are an important factor in comparing cost effectiveness of the different treatment options for couples seeking treatment after the male partner has undergone vasectomy. ${ }^{31}$

\section{Conclusion}

We have demonstrated that microsurgical 1-layer VV has excellent patency rates and is highly successful in enabling men who have had a vasectomy to renew paternity. We also found that preoperative and intraoperative factors are significantly associated with success and fertility status after VV. The presence of antisperm antibodies in the ejaculate significantly reduces the likelihood of establishing a pregnancy. Finally, we have established that measurement of the concentration of AG in ejaculate following VV is useful to predict patency and pregnancy results after microsurgical repair.

From the Division of Urology, Centre Hospitalier Universitaire de Québec (CHUQ), Laval University, Québec, Que.

This article has been peer reviewed.

Competing interests: None declared.

\section{References}

1. Mosher WD. Contraceptive practice in the United States, 1982-1988. Fam Plann Perspect 1990;22:198-205.

2. Gallen ME, Liskin L, Kak N. Men - new focus for family planning programs. Popul Rep J 1986;Nov-Dec:J889-919.

3. Cos LR, Valvo JR, Davis RS, et al. Vasovasostomy: current state of the art. Urology 1983;22:567-75.

4. Middleton $R G$, Henderson D. Vas deferens reanastomosis without splints and without magnification. J Urol 1978;1 19:763-4.

5. Urquhart-Hay D. A low power magnification technique for reanastomosis of the vas. $\mathrm{Br} J$ Urol 1981;53:466-9. 
Bolduc et al

6. Shessel FS, Lynne CM, Politano VA. Use of exteriorized stents in vasovasostomy. Urology 1981;17:163-5.

7. Silber SJ. Microscopic vasectomy reversal. Fertil Steril 1977;28:1191-202.

8. Belker AM, Thomas AJ Jr, Fuchs EF, et al. Results of 1,469 microsurgical vasectomy reversals by the Vasovasostomy Study Group. J Urol 1991;145:505-11.

9. Fuse H, Kimura H, Katayama T. Modified one-layer microsurgical vasovasostomy in vasectomized patients. Int Urol Nephrol 1995;27:451-6.

10. Matthews GJ, McGee KE, Goldstein M. Microsurgical reconstruction following failed vasectomy reversal. J Urol 1997;157:844-6.

11. Goldstein M, Li PS, Matthews GJ. Microsurgical vasovasostomy: the microdot technique of precision suture placement. J Urol 1998;159:188-90.

12. Jokelainen OS, Rintala E, Koskimies Al, et al. Vasovasostomy - a 15-year experience. Scand I Urol Nephrol 2001;35:132-5.

13. Holman CD, Wisniewski ZS, Semmens JB, et al. Population-based outcomes after 28,246 in-hospital vasectomies and 1,902 vasovasostomies in Western Australia. BJU Int 2000;86:1043-9.

14. Tremblay RR, Chapdelaine P, Mailhot J. alpha, 1-4-Glucosidase activity in human semen: variations with number and motility of spermatozoa. Fertil Steril 1979;31:592-3.

15. Tremblay RR, Paquin R, Thabet $M$. Nature of the residual alpha-1,4-glucosidase activity in the seminal plasma of vasectomized men. Enzyme 1985;34:33-8.

16. Guerin JF, Ali HB, Rollet J, et al. Alpha-glucosidase as a specific epididymal enzyme marker. Its validity for the etiologic diagnosis of azoospermia. J Androl 1986;7:156-62.

17 [WHO laboratory manual for the examination of human seminal fluid and the interaction between seminal fluid and cervix mucus]. [Article in Italian]. Ann Ist Super Sanita 1989;25(1 Suppl):1-67.

18. Chapdelaine P, Tremblay RR, Dube JY. P-Nitrophenol-alpha-D-glucopyranoside as substrate for measurement of maltase activity in human semen. Clin Chem 1978;24:208-11.

19. Cimino C, Barba G, Guastella $G$, et al. An ElISA for antisperm antibody detection in serum: comparison with TAT and SIT in serum, with MAR-test, immunobead-test and TAT in semen and with micro-SIT in cervical mucus. Acta Eur Fertil 1987;18:11-9.

20. Silber SJ. Sperm granuloma and reversibility of vasectomy. Lancet 1977;2:588-9.
21. Jarow JP, Budin RE, Dym M, et al. Quantitative pathologic changes in the human testis after vasectomy. A controlled study. N Engl J Med 1985;313:1252-6.

22. Silber SI. Epididymal extravasation following vasectomy as a cause for failure of vasectomy reversal. Fertil Steril 1979;31:309-15.

23. Linnet L. Clinical immunology of vasectomy and vasovasostomy. Urology 1983;22: 101-14.

24. Meinertz $\mathrm{H}$, Linnet L, Fogh-Andersen $\mathrm{P}$, et al. Antisperm antibodies and fertility after vasovasostomy: a follow-up study of 216 men. Fertil Steril 1990;54:315-21.

25. Matthews GJ, Schlegel PN, Goldstein M. Patency following microsurgical vasoepididymostomy and vasovasostomy: temporal considerations. J Urol 1995; 154:2070-3.

26. Donovan JF Jr, DiBaise M, Sparks AE, et al. Comparison of microscopic epididymal sperm aspiration and intracytoplasmic sperm injection/in-vitro fertilization with repeat microscopic reconstruction following vasectomy: is second attempt vas reversal worth the effort? Hum Reprod 1998;13:387-93.

27. Fox M. Failed vasectomy reversal: is a further attempt using microsurgery worthwhile? BJU Int 2000;86:474-8.

28. Cooper TG. Secretory proteins from the epididymis and their clinical relevance. Andrologia 1990;22:155-65.

29. Heidenreich A, Altmann P, Neubauer $S$, et al. [Microsurgical vasovasostomy in the age of modern reproduction medicine. A cost-benefit analysis]. [Arricle in German]. Urologe A 2000;39:240-5.

30. Deck AJ, Berger RE. Should vasectomy reversal be performed in men with older female partners? J Urol 2000;163:105-6.

31. Meng MV, Greene KL, Turek PJ. Surgery or assisted reproduction? A decision analysis of treatment costs in male infertility. J Urol 2005;174:1926-31.

Correspondence: Dr. Stéphane Bolduc, Division of Urology, Centre Hospitalier Universitaire de Québec (CHUQ, CHUL), 2705 Boul Laurier, R-1742, Québec QC GIV 4G2; sbolduc_2002@yahoo.ca 